\title{
Weakly Kronecker equivalent number fields
}

\author{
by \\ MANFRED LOCHTER (Saarbrücken)
}

1. Introduction. In [5], [6] W. Jehne has introduced and investigated the fundamental notion of Kronecker equivalence. Two extensions $K \mid k$ and $K^{\prime} \mid k$ of number fields are called Kronecker equivalent over $k\left(K \sim_{k} K^{\prime}\right)$ iff the sets $D(K \mid k)$ of all primes of $k$ which have a divisor of first degree in $K$ and $D\left(K^{\prime} \mid k\right)$ coincide up to at most finitely many exceptions (there are as a matter of fact no exceptions at all).

Kronecker equivalent fields have been studied by several authors (e.g. R. Guralnick, N. Klingen, Ch. E. Praeger, J. Saxl, V. Schulze), but only recently [12] we found a new characterization of Kronecker equivalence. Let $P(k)$ denote the set of finite primes of $k$. Then $K$ and $K^{\prime}$ are Kronecker equivalent over $k$ iff for all $\wp \in P(k)$,

$$
\mathbb{N} f_{1}+\ldots+\mathbb{N} f_{r}=\mathbb{N} f_{1}^{\prime}+\ldots+\mathbb{N} f_{s}^{\prime} .
$$

Here we assume $\wp$ to have decomposition type $\left(f_{1}, \ldots, f_{r}\right)$ in $K$, e.g. $\wp$ possesses exactly $r$ prime divisors $\mathcal{P}_{1}, \ldots, \mathcal{P}_{r}$ of degrees $f_{1}:=f_{K \mid k}\left(\mathcal{P}_{1}\right) \leq \ldots \leq$ $f_{r}:=f_{K \mid k}\left(\mathcal{P}_{r}\right)$ in $K$. Let $N \mid k$ be a Galois extension which contains $K$. $G=G(N \mid k)$ acts on the left cosets of $U=G(N \mid K)$ and for unramified $\wp \in P(k)$ and any Frobenius automorphism $\sigma$ corresponding to a divisor of $\wp$ in $N$ the following statements are equivalent:

(a) $\wp$ has decomposition type $\left(f_{1}, \ldots, f_{r}\right)$ in $K$.

(b) $\sigma$ acts on the left cosets of $U$ as a product of $r$ disjoint cycles of lengths $f_{1} \leq \ldots \leq f_{r}$ (the cycle type of $\sigma$ is $\left(f_{1}, \ldots, f_{r}\right)$ ).

From (1) we conclude that for all $\wp \in P(k)$,

$$
\operatorname{gcd}\left(f_{K \mid k}(\mathcal{P})|\mathcal{P} \in P(K) \wedge \mathcal{P}| \wp\right)=\operatorname{gcd}\left(f_{K^{\prime} \mid k}(\mathcal{P})\left|\mathcal{P} \in P\left(K^{\prime}\right) \wedge \mathcal{P}\right| \wp\right) .
$$

We call $K$ and $K^{\prime}$ weakly Kronecker equivalent over $k$ iff (2) holds for (almost) all $\wp \in P(k)$. A first interesting result on the notion of weak Kronecker equivalence is the following number theoretic theorem. 
THEOREM 1. $K$ and $K^{\prime}$ are weakly Kronecker equivalent over $k$ iff $\left(N_{K \mid k}\left(K^{*}\right): N_{K \mid k}\left(K^{*}\right) \cap N_{K^{\prime} \mid k}\left(K^{\prime *}\right)\right)$ and $\left(N_{K^{\prime} \mid k}\left(K^{\prime *}\right): N_{K \mid k}\left(K^{*}\right) \cap\right.$ $\left.N_{K^{\prime} \mid k}\left(K^{\prime *}\right)\right)$ are finite.

A proof of Theorem 1 was published in another paper $\left(^{*}\right)$. Theorem 1 together with the results of the present paper give some interesting class field theoretic theorems.

It is well known that abelian number fields are uniquely determined by their idele class norm groups. Theorem 1 (together with Theorem 10) shows that Galois extensions $K$ of $k$ are characterized - among all Galois extensions of $k$-by their group of global norms. Further, extensions of degree $n$ with group $A_{n>5}$ or $S_{n}$ are characterized among all extensions of $k$ by their group of global norms. Thus (see [22]) "almost all" extensions of algebraic number fields are characterized by their group of global norms. Although there is no description of the extensions of $k$ in terms of $k$, as given for abelian extensions by class field theory, the above results may be seen as a partial generalization of class field theory.

In addition weakly Kronecker equivalent fields (different from the ground field) contain the same Galois extensions of $k$ and their degrees are not coprime (Theorems 13, 14). The weak Kronecker class of the ground field is trivial.

The absence of exceptional primes allows us to find explicit bounds for the index $\left(N_{K \mid k}\left(K^{*}\right): N_{K \mid k}\left(K^{*}\right) \cap N_{K^{\prime} \mid k}\left(K^{\prime *}\right)\right)$ if it is finite. This bound involves class numbers, the order of the group of roots of unity contained in $K$, degrees and unit ranks.

But Kronecker equivalence has also attracted the interest of group theorists. Let $K \mid k$ and $K^{\prime} \mid k$ be number fields contained in a Galois extension $N \mid k$ and let $G=G(N \mid k), U=G(N \mid K)$ and $U^{\prime}=G\left(N \mid K^{\prime}\right)$ be the respective Galois groups. Then $K$ and $K^{\prime}$ are Kronecker equivalent over $k$ iff

$$
U^{G}=\bigcup_{\sigma \in G} U^{\sigma}=U^{\prime G},
$$

e.g. iff $U$ and $U^{\prime}$ are elementwise conjugate. This covering property has been intensively studied by Ch. E. Praeger [15]-[17], J. Saxl [18] and others.

Since it is easier to look at Sylow $p$-subgroups $U_{p}$ of $G$, it is interesting to investigate the following covering property:

$$
\bigcup_{p \mid \# G} U_{p}^{G}=\bigcup_{p \mid \# G} U_{p}^{\prime G}
$$

It is an amazing observation that $K \mid k$ and $K^{\prime} \mid k$ are weakly Kronecker equivalent over $k$ iff (4) holds. This is a second reason to study weakly Kronecker

$\left(^{*}\right)$ Weakly Kronecker equivalent number fields and global norms, this volume, pp. 105121. 
equivalent fields. A third incentive may be seen in the fact that all important theorems for Kronecker equivalent fields also hold for weakly Kronecker equivalent fields. The major aim of this paper is to establish these theorems. The proofs needed here, however, are quite different from the original ones and use group theoretical theorems, which are based upon the classification of finite simple groups. But one question remains open: for which groups $G$ with subgroups $U, U^{\prime}$ does $\bigcup_{p \mid \# G} U_{p}^{G}=\bigcup_{p \mid \# G} U_{p}^{\prime G}$ imply $U^{G}=U^{\prime G}$ ?

\section{Group theoretical and representation theoretical description}

Definition 2. Let $\pi$ be a set of prime numbers. Two extensions $K \mid k$ and $K^{\prime} \mid k$ are called $\pi$-Kronecker equivalent $\left(K \sim_{\pi, k} K^{\prime}\right)$ over $k$ iff for almost all $\wp \in P(k)$ and for all $p \in \pi$,

$$
\begin{aligned}
\min \left\{\nu_{p}\left(f_{K \mid k}(\mathcal{P})\right) \mid \mathcal{P} \in P(K)\right. & \wedge \mathcal{P} \mid \wp\} \\
& =\min \left\{\nu_{p}\left(f_{K^{\prime} \mid k}(\mathcal{P})\right)\left|\mathcal{P} \in P\left(K^{\prime}\right) \wedge \mathcal{P}\right| \wp\right\} .
\end{aligned}
$$

Here $\nu_{p}$ denotes the usual $p$-adic valuation. $K$ and $K^{\prime}$ are called weakly Kronecker equivalent over $k\left(K \sim_{s, k} K^{\prime}\right)$ if $\pi$ is the set $\mathbb{P}$ of all prime numbers.

Let $G$ be a finite group. For a subgroup $U$ of $G, \Phi_{U}=1_{U}^{G}$ is the character of $G$ induced from the trivial representation of $U$. Given a prime divisor of $\# G$, we define $n_{p}$ by

$$
\# G=p^{\nu_{p}(\# G)} \cdot n_{p} .
$$

Further, let $\left\{p_{1}, \ldots, p_{l}\right\}$ be the set of prime divisors of $\# G$. We identify $\mathbb{N}^{\left\{p_{1}, \ldots, p_{l}\right\}}$ with $\mathbb{N}^{l}$. For $\underline{i} \in \mathbb{N}^{l}$ we define

$$
\Psi_{U, \pi, \underline{i}}: G \rightarrow \mathbb{N}, \quad \sigma \mapsto \prod_{\pi \ni p \mid \# G} \Phi_{U}\left(\sigma^{n_{p} \cdot p^{i_{p}}}\right) .
$$

By [20, Exc. 9.3], $\Psi_{U, \pi, \underline{i}}$ is a virtual character of $G$. We abbreviate $\Psi_{U, \mathbb{P}, \underline{i}}=$ $\Psi_{U, \underline{i}}, \Psi_{U, \pi, \underline{0}}=\Psi_{U, \pi}$ and $\Psi_{U}=\Psi_{U, \underline{0}}$. For $n \in \mathbb{N}, \psi^{n}\left(\Phi_{U}\right)$ denotes the virtual character $\sigma \mapsto \Phi_{U}\left(\sigma^{n}\right)$.

The next theorem contains as a special case descriptions of $\pi$-Kronecker equivalence and of weak Kronecker equivalence.

TheOrem 3. Let $K \mid k$ and $K^{\prime} \mid k$ be contained in a Galois extension $N \mid k$ of $k$ with group $G$. For a prime divisor $p$ of $\# G, K_{p}$ denotes the fixed field of a Sylow p-subgroup $U_{p}$ of $U=G(N \mid K)$. Similar notation is adopted for $K^{\prime}$. Given a set $\pi$ of prime numbers the following conditions are equivalent:

(i) There is a subset $M \subset P(k)$ of Dirichlet density 0 so that for all $\wp \in P(k) \backslash M$ and for all $p \in \pi$,

$$
\begin{aligned}
\min \left\{\nu_{p}\left(f_{K \mid k}(\mathcal{P})\right) \mid \mathcal{P} \in\right. & P(K) \wedge \mathcal{P} \mid \wp\}=0 \\
& \Rightarrow \min \left\{\nu_{p}\left(f_{K^{\prime} \mid k}(\mathcal{P})\right)\left|\mathcal{P} \in P\left(K^{\prime}\right) \wedge \mathcal{P}\right| \wp\right\}=0 .
\end{aligned}
$$


(ii) For all $\wp \in P(k)$ and for all $p \in \pi$,

$$
\begin{aligned}
& \min \left\{\nu_{p}\left(f_{K \mid k}(\mathcal{P})\right)|\mathcal{P} \in P(K) \wedge \mathcal{P}| \wp\right\} \\
& \geq \min \left\{\nu_{p}\left(f_{K^{\prime} \mid k}(\mathcal{P})\right)\left|\mathcal{P} \in P\left(K^{\prime}\right) \wedge \mathcal{P}\right| \wp\right\} . \\
& \text { (iii) } \bigcup_{p \in \pi} U_{p}^{G} \subset \bigcup_{p \in \pi} U_{p}^{\prime G} \text {. } \\
& \text { (iv) } \bigwedge_{p \in \pi} D\left(K_{p} \mid k\right) \subset D\left(K_{p}^{\prime} \mid k\right) \text {. } \\
& \text { (v) } \bigwedge_{\sigma \in G}\left[\bigwedge_{p \in \pi} \Phi_{U}\left(\sigma^{n_{p}}\right)>0 \Rightarrow \bigwedge_{p \in \pi} \Phi_{U^{\prime}}\left(\sigma^{n_{p}}\right)>0\right] \text {. } \\
& \text { (vi) For all } \phi=\sum n_{\sigma} \sigma \in \mathbb{Q}[G] \text { with } n_{\sigma} \geq 0 \text { and for all } \underline{i} \in \mathbb{N}^{l} \text {, } \\
& \Psi_{U, \pi, \underline{i}}(\phi)>0 \Rightarrow \Psi_{U^{\prime}, \pi, \underline{i}}(\phi)>0 \text {. } \\
& \text { (vii) } \bigwedge_{\wp \in P(k)}\left[\prod_{\substack{p \in \pi \\
p \mid \# G}}\left(\sum_{f_{i} \mid n_{p}} f_{i}\right)>0 \Rightarrow \prod_{\substack{p \in \pi \\
p \mid \# G}}\left(\sum_{f_{i}^{\prime} \mid n_{p}} f_{i}^{\prime}\right)>0\right] \text {. }
\end{aligned}
$$

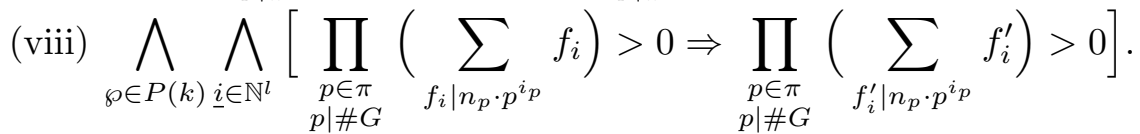

REMARK 4. (i) In Theorem 3 it is possible to replace $\Psi_{U, \pi, i}$ by $\widehat{\Psi}_{U, \pi, \underline{i}}=\prod_{\pi \ni p \mid \# G} \Phi_{U_{p}}\left(\sigma^{n_{p} \cdot p^{i_{p}}}\right)$ and to replace $\prod_{\pi \ni p \mid \# G}\left(\sum_{f_{i} \mid n_{p} \cdot p^{i_{p}}} f_{i}\right)$ by $\prod_{\pi \ni p \mid \# G}\left(\sum_{f_{i, p} \mid n_{p} \cdot p^{i p}} f_{i, p}\right)$, where $f_{i, p}$ runs through the residue degrees of the divisors of $\wp$ in $K_{p}$.

(ii) In Theorem 3 it is sufficient to consider only the primes $p \in \pi$ dividing $\# G$.

The proof of Remark 4 is left to the reader.

Proof of Theorem 3. Given $\wp \in P(k)$ and a divisor $\mathcal{P}$ of $\wp$ in $N$ we define

$$
F_{N \mid k}(\mathcal{P})=\frac{1}{e_{N \mid k}(\mathcal{P})} \sum_{\tau \in \mathcal{T}_{N \mid k}(\mathcal{P})} \sigma \tau,
$$

where $\mathcal{T}_{N \mid k}(\mathcal{P})$ is the inertia group of $\mathcal{P} \mid \wp, e_{N \mid k}(\mathcal{P})$ the ramification index of $\mathcal{P} \mid \wp$ and $\sigma$ any of the Frobenius automorphisms corresponding to $\mathcal{P}$. If $\left.\mathcal{P}\right|_{\wp}$ is unramified then $F_{N \mid k}(\mathcal{P})$ is the Frobenius automorphism corresponding to $\mathcal{P} \mid \wp$. In [12] we calculated

$$
\bigwedge_{j \in \mathbb{N}_{+}} \Phi_{U}\left(F_{N \mid k}(\mathcal{P})^{j}\right)=\sum_{f_{i} \mid j} f_{i}
$$

This fact is fundamental for the proof of Theorem 3. The equivalence of (iii), (iv) and (v) is well known (cf. [7]).

$(\mathrm{vi}) \Rightarrow(\mathrm{v})$ is trivial, namely just specialization $\underline{i}=\underline{0}$ and $\phi=\sigma$. 
(iii) $\Rightarrow$ (vi). For $\sigma \in G$ we see that

$$
\begin{aligned}
\Psi_{U, \pi, \underline{i}}(\sigma)>0 & \Leftrightarrow \prod_{\substack{p \in \pi \\
p \mid \# G}} \Phi_{U}\left(\sigma^{n_{p} \cdot p^{i_{p}}}\right)>0 \\
& \Leftrightarrow \bigwedge_{p \in \pi} \Phi_{U}\left(\sigma^{n_{p} \cdot p^{i_{p}}}\right)>0 \Leftrightarrow \bigwedge_{p \in \pi} \sigma^{n_{p} \cdot p^{i_{p}}} \in U_{p}^{G} \\
& \Rightarrow \bigwedge_{p \in \pi} \sigma^{n_{p} \cdot p^{i_{p}}} \in U_{p}^{\prime G} \Leftrightarrow \Psi_{U^{\prime}, \pi, \underline{i}}(\sigma)>0 .
\end{aligned}
$$

For $\phi=\sum n_{\sigma} \sigma \in \mathbb{Q}[G]$ with coefficients $n_{\sigma} \geq 0$ we now conclude

$$
\begin{aligned}
\Psi_{U, \pi, \underline{i}}(\phi)>0 & \Leftrightarrow \bigvee_{\sigma} n_{\sigma} \Psi_{U, \pi, \underline{i}}(\sigma)>0 \\
& \Rightarrow \bigvee_{\sigma} n_{\sigma} \Psi_{U^{\prime}, \pi, \underline{i}}(\sigma)>0 \Rightarrow \Psi_{U^{\prime}, \pi, \underline{i}}(\phi)>0
\end{aligned}
$$

(v) $\Rightarrow$ (viii). Let $\phi=\sum n_{\sigma} \sigma \in \mathbb{Q}[G], n_{\sigma} \geq 0, p \in \pi$ and assume $\psi^{n_{p}}\left(\Phi_{U}\right)(\phi)>0$. Since $(\mathrm{v}) \Leftrightarrow$ (iii) we have

$$
\bigvee_{\sigma} n_{\sigma} \cdot \Phi_{U}\left(\sigma^{n_{p}}\right)>0 \Rightarrow \bigvee_{\sigma} n_{\sigma} \cdot \Phi_{U^{\prime}}\left(\sigma^{n_{p}}\right)>0 \Rightarrow \psi^{n_{p}}\left(\Phi_{U^{\prime}}\right)(\phi)>0
$$

Now the proof of (viii) is simple for unramified $\wp$. But the following Theorem 5 allows us to give a proof which also works in the ramified case.

$$
\begin{aligned}
& \prod_{\substack{p \in \pi \\
p \mid \# G}}\left(\sum_{f_{i} \mid n_{p} \cdot p^{i_{p}}} f_{i}\right)>0 \stackrel{(6)}{\Longleftrightarrow} \bigwedge_{\substack{p \in \pi \\
p \mid \# G}} \Phi_{U}\left(F_{N \mid k}(\mathcal{P})^{n_{p} \cdot p^{i_{p}}}\right)>0 \\
& \stackrel{\text { Thm. }}{\Longleftrightarrow} \bigwedge_{\substack{p \in \pi \\
p \mid \# G}} \psi^{n_{p}}\left(\Phi_{U}\right)\left(F_{N \mid k}(\mathcal{P})^{n_{p} \cdot p^{i_{p}}}\right)>0 \\
& \Rightarrow \bigwedge_{\substack{p \in \pi \\
p \mid \# G}} \psi^{n_{p}}\left(\Phi_{U^{\prime}}\right)\left(F_{N \mid k}(\mathcal{P})^{n_{p} \cdot p^{i p}}\right)>0 \\
& \stackrel{\text { Thm. }}{\Longleftrightarrow} \bigwedge_{\substack{p \in \pi \\
p \mid \# G}} \Phi_{U^{\prime}}\left(F_{N \mid k}(\mathcal{P})^{n_{p} \cdot p^{i_{p}}}\right)>0 \\
& \stackrel{(6)}{\Longleftrightarrow} \prod_{\substack{p \in \pi \\
p \mid \# G}}\left(\sum_{f_{i}^{\prime} \mid n_{p} \cdot p^{i} p} f_{i}^{\prime}\right)>0 .
\end{aligned}
$$

(viii) $\Rightarrow$ (ii). For $\wp \in P(k)$ we define

$$
i_{p}= \begin{cases}\min \left\{\nu_{p}\left(f_{i}\right)\right\} & \text { if } p \in \pi, \\ 0 & \text { otherwise. }\end{cases}
$$


Then

$$
\begin{aligned}
\prod_{\substack{p \in \pi \\
p \mid \# G}}\left(\sum_{f_{i} \mid n_{p} \cdot p^{i_{p}}} f_{i}\right)>0 & \stackrel{\text { (viii) }}{\Longrightarrow} \prod_{\substack{p \in \pi \\
p \mid \# G}}\left(\sum_{f_{i}^{\prime} \mid n_{p} \cdot p^{i_{p}}} f_{i}^{\prime}\right)>0 \\
& \Rightarrow \bigwedge_{\substack{p \in \pi \\
p \mid \# G}} \bigvee_{i}^{\prime} \nu_{p}\left(f_{i}^{\prime}\right) \leq i_{p} \Rightarrow \bigwedge_{\substack{p \in \pi \\
p \mid \# G}} \min \left\{\nu_{p}\left(f_{i}^{\prime}\right)\right\} \leq i_{p} .
\end{aligned}
$$

(ii) $\Rightarrow$ (i). Trivial.

(i) $\Rightarrow$ (iii). Let $\sigma \in U_{p}^{G}, p \in \pi$ and take $\wp \in \pi$ unramified such that $\sigma=F_{N \mid k}\left(\mathcal{P}_{0}\right)$ for a divisor of $\wp$ in $N$. Then $\min \left\{\nu_{p}\left(f_{K^{\prime} \mid k}(\mathcal{P})\right) \mid \mathcal{P} \in\right.$ $\left.P\left(K^{\prime}\right) \wedge \mathcal{P} \mid \wp\right\}=0$. Since $\sigma \in G$ has $p$-power order, at least one of the residue degrees is 1 . Hence $\sigma \in U_{p}^{\prime G}$.

(vii) $\Leftrightarrow(\mathrm{i})$. We have

$$
\sum_{f_{i} \mid n_{p}} f_{i}>0 \Leftrightarrow \min \left\{\nu_{p}\left(f_{K \mid k}(\mathcal{P})\right)|\mathcal{P} \in P(K) \wedge \mathcal{P}| \wp\right\}=0 .
$$

The importance of Theorem 3 consists in a criterion for weak Kronecker equivalence, which we formulate explicitly for the convenience of the reader:

THEOREM $3^{\prime}$. The following statements are equivalent for $K, K^{\prime}$ :

(i) $K$ and $K^{\prime}$ are weakly Kronecker equivalent over $k$.

(ii) $T F(K \mid k):=\left\{\wp \in P(k) \mid \operatorname{gcd}\left(f_{K \mid k}(\mathcal{P})|\mathcal{P} \in P(K) \wedge \mathcal{P}| \wp\right)=1\right\}=$ $T F\left(K^{\prime} \mid k\right)$.

(iii) $\bigcup_{p \mid \# G} U_{p}^{G}=\bigcup_{p \mid \# G} U_{p}^{\prime G}$.

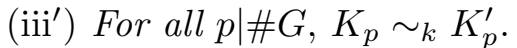

(iv) $\Psi_{U}$ and $\Psi_{U^{\prime}}$ have the same zeroes on $G$.

Thus $T F(K \mid k)$ is an analogue of the Kronecker set $D(K \mid k)$ which is studied for Kronecker equivalent fields. Note that again there are no exceptions.

Theorem 5. We maintain the notation of Theorem 3. For a prime divisor $p$ of $\# G, i_{p} \in \mathbb{N}$, a prime $\wp \in P(k)$ and a divisor $\mathcal{P} \in P(N)$ of $\wp$,

$$
\begin{aligned}
\Phi_{U}\left(F_{N \mid k}(\mathcal{P})^{n_{p} \cdot p^{i_{p}}}\right) & \leq \psi^{n_{p}}\left(\Phi_{U}\right)\left(F_{N \mid k}(\mathcal{P})^{n_{p} \cdot p^{i_{p}}}\right) \\
& \leq e_{N \mid k}(\mathcal{P}) \cdot \Phi_{U}\left(F_{N \mid k}(\mathcal{P})^{n_{p} \cdot p^{i_{p}}}\right) .
\end{aligned}
$$

Proof. First we note that for arbitrary $m \in \mathbb{N}_{+}$,

$$
F_{N \mid k}(\mathcal{P})^{m} \stackrel{(5)}{=}\left(\frac{1}{e_{N \mid k}(\mathcal{P})} \sum_{\tau \in \mathcal{T}_{N \mid k}(\mathcal{P})} \sigma \tau\right)^{m}=\frac{1}{e_{N \mid k}(\mathcal{P})} \sum_{\tau \in \mathcal{T}_{N \mid k}(\mathcal{P})} \sigma^{m} \tau .
$$


With (7) we conclude

$$
\begin{aligned}
\Phi_{U}\left(F_{N \mid k}(\mathcal{P})^{n_{p} \cdot p^{i_{p}}}\right) & =\Phi_{U}\left(\frac{1}{e_{N \mid k}(\mathcal{P})} \sum_{\tau \in \mathcal{T}_{N \mid k}(\mathcal{P})} \sigma^{n_{p} \cdot p^{i_{p}}} \tau\right) \\
& =\frac{1}{e_{N \mid k}(\mathcal{P})} \sum_{\tau \in \mathcal{T}_{N \mid k}(\mathcal{P})} \Phi_{U}\left(\sigma^{n_{p} \cdot p^{i_{p}}} \tau\right) \\
& \leq \frac{1}{e_{N \mid k}(\mathcal{P})} \sum_{\tau \in \mathcal{T}_{N \mid k}(\mathcal{P})} \Phi_{U}\left(\left(\sigma^{n_{p} \cdot p^{i_{p}}} \tau\right)^{n_{p}}\right) \\
& =\Phi_{U}\left(\frac{1}{e_{N \mid k}(\mathcal{P})} \sum_{\tau \in \mathcal{T}_{N \mid k}(\mathcal{P})}\left(\sigma^{n_{p} \cdot p^{i_{p}}} \tau\right)^{n_{p}}\right) \\
& =\psi^{n_{p}}\left(\Phi_{U}\right)\left(F_{N \mid k}(\mathcal{P})^{n_{p} \cdot p^{i_{p}}}\right) \\
& \leq \Phi_{U}\left(\frac{1}{e_{N \mid k}(\mathcal{P})^{n_{p}-1}}\left(\sum_{\tau \in \mathcal{T}_{N \mid k}(\mathcal{P})} \sigma^{n_{p} \cdot p^{i_{p}}} \tau\right)^{n_{p}}\right) \\
& \stackrel{(7)}{=} \Phi_{U}\left(e_{N \mid k}(\mathcal{P}) F_{N \mid k}(\mathcal{P})^{n_{p}^{2} \cdot p^{i_{p}}}\right) \\
& \stackrel{(5)}{=} e_{N \mid k}(\mathcal{P}) \sum_{f_{i} \mid n_{p}^{2} \cdot p^{i_{p}}} f_{i} \\
& =e_{N \mid k}(\mathcal{P}) \sum_{f_{i} \mid n_{p} \cdot p^{i_{p}}} f_{i} \\
& =e_{N \mid k}(\mathcal{P}) \Phi_{U}\left(F_{N \mid k}(\mathcal{P})^{n_{p} \cdot p^{i_{p}}}\right) .
\end{aligned}
$$

Here (8) holds, because every fixed point of $\eta \in G$ on $G / U$ is a fixed point of $\eta^{n_{p}}$. (11) follows from $f_{i}\left|n_{p} \cdot p^{i_{p}} \Leftrightarrow f_{i}\right| n_{p}^{2} \cdot p^{i_{p}}$. Obviously

$$
(8) \leq \max _{\tau \in \mathcal{T}_{N \mid k}(\mathcal{P})} \Phi_{U}\left(\sigma^{n_{p}^{2} p^{i_{p}}} \tau\right) .
$$

From (7) we conclude $(10)=\Phi_{U}\left(\sum_{\tau \in \mathcal{T}_{N \mid k}(\mathcal{P})} \sigma^{n_{p}^{2} p^{i_{p}}} \tau\right)$. Hence $(8) \leq(10)$.

The exact conditions for equality to hold in Theorem 5 are unknown. We remark that the proof of Theorem 3 can also be accomplished by the following fact, which holds for every $p$ :

$$
\begin{aligned}
\min \left\{\nu_{p}\left(f_{K \mid k}(\mathcal{P})\right) \mid \mathcal{P}\right. & \in P(K) \wedge \mathcal{P} \mid \wp\} \\
& =\min \left\{\nu_{p}\left(f_{K_{p} \mid k}(\mathcal{P})\right)\left|\mathcal{P} \in P\left(K_{p}\right) \wedge \mathcal{P}\right| \wp\right\} .
\end{aligned}
$$

The proof of (12) is left to the reader.

Now we are prepared to describe the set $T F(K \mid k)$ by the zeroes of a virtual character. 
TheOREM 6. Let $N \mid k$ be a Galois extension and $K$ be the fixed field of a subgroup $U$ of $G(N \mid k)$. Let $\left\{p_{1}, \ldots, p_{l}\right\}$ be the set of prime divisors of \#G. For $\underline{i}, \underline{j} \in \mathbb{N}^{l}=\mathbb{N}^{\left\{p_{1}, \ldots, p_{l}\right\}}$ we define $\underline{i} \leq \underline{j} \Leftrightarrow \bigwedge_{p \mid \# G} i_{p} \leq j_{p}$. Then

$$
\begin{aligned}
& \left\{\wp \in P(k) \mid \operatorname{gcd}\left(f_{1}, \ldots, f_{r}\right)=\prod_{p \mid \# G} p^{i_{p}}\right\} \\
& =\left\{\wp \in P(k) \mid \underline{i}=\min \left\{\underline{j} \in \mathbb{N}^{l} \mid \bigwedge_{p \mid \# G} \Phi_{U}\left(F_{N \mid k}(\mathcal{P})^{n_{p} \cdot p^{j_{p}}}\right)>0\right.\right. \\
& \quad \text { for one }(\text { all }) \operatorname{divisor}(s) \mathcal{P} \text { of } \wp \text { in } N\}\} .
\end{aligned}
$$

Proof. For all $p \mid \# G$ and for all $\wp \in P(k)$

$$
\begin{aligned}
i_{p} & =\min \left\{\nu_{p}\left(f_{1}\right), \ldots, \nu_{p}\left(f_{r}\right)\right\} \\
\Leftrightarrow \quad i_{p} & =\min \left\{j_{p} \mid \sum_{f_{i} \mid n_{p} \cdot p^{j_{p}}} f_{i}>0\right\} \\
\stackrel{(5)}{\Longleftrightarrow} i_{p} & =\min \left\{j_{p} \mid \Phi_{U}\left(F_{N \mid k}(\mathcal{P})^{n_{p} \cdot p^{j_{p}}}\right)>0\right\} \\
\stackrel{\text { Thm. }}{\Longleftrightarrow} i_{p} & =\min \left\{j_{p} \mid \psi^{n_{p}}\left(\Phi_{U}\right)\left(F_{N \mid k}(\mathcal{P})^{n_{p} \cdot p^{j_{p}}}\right)>0\right\} .
\end{aligned}
$$

3. Application to class groups. For an extension $K \mid k$ of number fields we investigate the norm homomorphism $\mathcal{N}_{K \mid k}: \mathrm{Cl}(K) \rightarrow \mathrm{Cl}(k)$ on class groups. $\mathcal{N}_{K \mid k}$ is induced from the norm map $I_{K} \rightarrow I_{k}$ on fractional ideals. On the set $P(K)$ of maximal ideals $\mathcal{N}_{K \mid k}$ is defined by $\mathcal{P} \mapsto \wp^{f_{K \mid k}(\mathcal{P})}$, where $\wp$ is the prime ideal below $\mathcal{P}$, and extended to $I_{K}$ by linearity.

Theorem 7. Let $K$ and $K^{\prime}$ be weakly Kronecker equivalent over $k$. Then

$$
\mathcal{N}_{K \mid k}(\mathrm{Cl}(K))=\mathcal{N}_{K^{\prime} \mid k}\left(\mathrm{Cl}\left(K^{\prime}\right)\right) .
$$

Proof. Obviously $\mathcal{N}_{K \mid k}\left(I_{K}\right)=\left\langle\wp^{\operatorname{gcd}\left(f_{K \mid k}(\mathcal{P})|\mathcal{P} \in P(K) \wedge \mathcal{P}| \wp\right)} \mid \wp \in P(k)\right\rangle$. By Theorem $3^{\prime}, \mathcal{N}_{K \mid k}\left(I_{K}\right)=\mathcal{N}_{K^{\prime} \mid k}\left(I_{K^{\prime}}\right)$. Since the norm respects principal ideals we are done.

4. Variations and extensions of weak Kronecker equivalence. For the following sections the deep and widely unknown group theoretical result of Fein, Kantor and Schacher is of fundamental significance:

TheOREM 8. Let $G$ be a finite group acting transitively on a finite set $X$ with $\# X>1$. Then $G$ contains an element of prime power order which acts without fixed points.

Pr o of. See [1].

Two number fields are Kronecker equivalent iff the characters $\Phi_{U}$ and $\Phi_{U^{\prime}}$ possess the same zeroes; they are called arithmetically equivalent [14] iff 
$\Phi_{U}=\Phi_{U^{\prime}}$. By Theorem $3^{\prime}$, two number fields are weakly Kronecker equivalent iff $\Psi_{U}$ and $\Psi_{U^{\prime}}$ have the same zeroes. This suggests investigating the condition $\Psi_{U}=\Psi_{U^{\prime}}$.

Theorem 9. Let $K$ and $K^{\prime}$ be subfields of a Galois extension $N \mid k$ with groups $U=G(N \mid K)$ and $U^{\prime}=G\left(N \mid K^{\prime}\right)$. Let $\left\{p_{1}, \ldots, p_{l}\right\}$ be the prime divisors of the order of $G=G(N \mid k)$.

(a) The following statements are equivalent:

(i) $\Psi_{U}=\Psi_{U^{\prime}}$.

(ii) There is a subset $M \subset P(k)$ of Dirichlet density 0, so that for all $\wp \in P(k) \backslash M$ and for all $p \mid \# G$,

$$
\sum_{p \mid f_{i}} f_{i}=\sum_{p \mid f_{i}^{\prime}} f_{i}^{\prime}
$$

(iii) For almost all $\wp \in P(k)$, for all $p \mid \# G$ and for all $\underline{i} \in \mathbb{N}^{l}$,

$$
\sum_{f_{i} \mid n_{p} \cdot p^{i_{p}}} f_{i}=\sum_{f_{i}^{\prime} \mid n_{p} \cdot p^{i_{p}}} f_{i}^{\prime}
$$

(b) If one of the equivalent conditions of (a) holds, then $K$ and $K^{\prime}$ have the same degree over $k:(K: k)=\left(K^{\prime}: k\right)$, and the same normal closure over $k: \widetilde{K}=\widetilde{K^{\prime}}$.

The properties mentioned in (b) are typical of arithmetically equivalent fields. Together with the above remarks this suggests speaking of weakly arithmetically equivalent fields.

One should also note that the condition $\Psi_{U}=\Psi_{U^{\prime}}$ is independent of the Galois extension $N$ containing $K$ and $K^{\prime}$.

Proof of Theorem 9. Since $\Psi_{U}(1)=(G: U)^{l}$ the equality $\Psi_{U}=\Psi_{U^{\prime}}$ implies $\# U=\# U^{\prime}$. Further,

$$
\Psi_{U}=\Psi_{U^{\prime}} \Leftrightarrow \bigwedge_{p \mid \# G} \psi^{n_{p}}\left(\Phi_{U}\right)=\psi^{n_{p}}\left(\Phi_{U^{\prime}}\right)
$$

Proof of (13). " $\Rightarrow$ " For $\sigma \in G_{p}^{G}$ we calculate

$$
(G: U)^{l-1} \cdot \Phi_{U}\left(\sigma^{n_{p}}\right)=\Phi_{U^{\prime}}\left(\sigma^{n_{p}}\right) \cdot(G: U)^{l-1} .
$$

Thus $\Phi_{U}$ and $\Phi_{U^{\prime}}$ coincide on $G_{p}^{G}$. For arbitrary $\sigma$ we immediately see

$$
\psi^{n_{p}}\left(\Phi_{U}\right)(\sigma)=\Phi_{U}\left(\sigma^{n_{p}}\right)=\Phi_{U^{\prime}}\left(\sigma^{n_{p}}\right)=\psi^{n_{p}}\left(\Phi_{U^{\prime}}\right)(\sigma) .
$$

The converse holds by definition.

(i) $\Leftrightarrow($ iii). Let $\wp \in P(k)$ be unramified and $\mathcal{P} \in P(N)$ a divisor of $\wp$ in $N$. The claim follows from (5) and (13).

(ii) $\Rightarrow$ (i). (ii) implies $(K: k)=\left(K^{\prime}: k\right)$. We may assume $(K: k)>1$. The group $G$ acts transitively on the set $G / U$. By the theorem of Fein, 
Kantor and Schacher (Theorem 8) there is $\sigma \in G$ of prime power order $p^{i}$ such that $\sigma$ acts without fixed points. Take any unramified $\wp \in P(k)$ with $\sigma=F_{N \mid k}(\mathcal{P})$ for a divisor $\mathcal{P} \in P(N)$ of $\wp$. Then

$$
(K: k)=\sum f_{i}=\sum_{p \mid f_{i}} f_{i}=\sum_{p \mid f_{i}^{\prime}} f_{i}^{\prime} \leq\left(K^{\prime}: k\right) .
$$

By symmetry $(K: k)=\left(K^{\prime}: k\right)$. Hence $\sum_{f_{i} \mid n_{p}} f_{i}=\sum_{f_{i}^{\prime} \mid n_{p}} f_{i}^{\prime}$ for almost all $\wp$. This gives $\Psi_{U}=\Psi_{U^{\prime}}$.

(iii) $\Rightarrow$ (ii). From (iii) it follows that $\# U=\# U^{\prime}$; hence we can use (iii) with $\underline{i}=\underline{0}$ in order to prove (ii).

(b) Let $U_{G}=\bigcap_{\sigma \in G} U^{\sigma}$. We already know $\operatorname{gcd}\left(n_{p_{1}}, \ldots, n_{p_{l}}\right)=1, \Psi_{U}=$ $\Psi_{U^{\prime}}$ and $\# U=\# U^{\prime}$. We calculate:

$$
\Psi_{U}(\sigma)=(G: U)^{l} \Leftrightarrow \bigwedge_{p \mid \# G} \sigma^{n_{p}} \in U_{G} \Leftrightarrow \sigma \in U_{G} .
$$

We only mention that the condition $\widehat{\Psi}_{U}=\widehat{\Psi}_{U^{\prime}}$ (cf. Remark 4) can also be investigated. The resulting theorem is obvious. In particular, $\widehat{\Psi}_{U}=\widehat{\Psi}_{U^{\prime}}$ implies $(K: k)=\left(K^{\prime}: k\right)$ and $\widetilde{K}=\widetilde{K^{\prime}}$.

It is also possible to extend the definition of weak Kronecker equivalence to $m$-tuples of fields. Let $K_{1}\left|k, \ldots, K_{m}\right| k, K_{1}^{\prime}, \ldots, K_{n}^{\prime} \mid k$ be number fields. The tuples $\left(K_{1}, \ldots, K_{m}\right)$ and $\left(K_{1}^{\prime}, \ldots, K_{n}^{\prime}\right)$ are called weakly Kronecker equivalent over $k$ if

$$
\begin{aligned}
\min \left\{\nu_{p}\left(f_{K_{i} \mid k}(\mathcal{P})\right) \mid i \in\{1, \ldots, m\}\right. & \left.\wedge \mathcal{P} \in P\left(K_{i}\right) \wedge \mathcal{P} \mid \wp\right\} \\
=\min \left\{\nu_{p}\left(f_{K_{i}^{\prime} \mid k}(\mathcal{P})\right) \mid i \in\{1, \ldots, n\}\right. & \left.\wedge \mathcal{P} \in P\left(K_{i}^{\prime}\right) \wedge \mathcal{P} \mid \wp\right\}
\end{aligned}
$$

holds for all prime numbers $p$ and for (almost) all $\wp \in P(k)$. Then it is easy to formulate analogues of Theorems 3 and 7 (cf. [11]).

5. Properties of $\pi$-Kronecker equivalence. We start with a reformulation of the theorem of M. Bauer [13].

TheOREm 10. Let $N \mid k$ be a Galois extension and $K \mid k$ an arbitrary extension with

$$
T F(N \mid k) ذ T F(K \mid k) \text {. }
$$

Then $K \supset N$.

Here $\dot{C}$ means "contained up to at most finitely many exceptions." See also Theorem $3^{\prime}$.

Proof. We can apply Bauer's theorem, since $T F(N \mid k) \doteq D(N \mid k)$ and $T F(K \mid k) \supset D(K \mid k)$. 
TheOREM 11. Let $K \mid k$ and $K^{\prime} \mid k$ be weakly Kronecker equivalent over $k$. Then $K$ and $K^{\prime}$ contain the same Galois extensions of $k$. In particular, the groups of roots of unity contained in $K$ and in $K^{\prime}$ coincide.

Proof. Choose a Galois extension $N \mid k$ which contains $K$ and $K^{\prime}$. Let $U=G(N \mid K), U^{\prime}=G\left(N \mid K^{\prime}\right)$ and $G=G(N \mid k)$. We consider a normal subgroup $H \triangleleft G$ with $H \supset U$. For every $p \mid \# G$ we choose Sylow $p$-subgroups $U_{p}, U_{p}^{\prime}$. Then

$$
H=H^{G} \supset \bigcup_{\sigma \in G} U_{p}^{\sigma}=U_{p}^{G}=U_{p}^{\prime G} \supset U_{p}^{\prime}
$$

Hence $H \supset U^{\prime}$.

The next two theorems generalize the Reduction Theorem and the Transition Theorem of W. Jehne [5].

THEOREM 12. Given a set $\pi$ of prime numbers, the following assertions hold:

(a) $K \sim_{\pi, k} K^{\prime} \Rightarrow K \sim_{\pi, k} K^{\prime} \cap \widetilde{K} \sim_{\pi, k} K \cap \widetilde{K^{\prime}}$.

(b) The minimal fields in a $\pi$-Kronecker class have the same normal closure over $k$; there are only finitely many minimal fields.

Proof. (b) is a simple consequence of (a).

(a) We choose a Galois extension $N \mid k$ containing $\widetilde{K}$ and $K^{\prime}$ and define $G=G(N \mid k), V=G\left(N \mid \widetilde{K} \cap K^{\prime}\right), U=G(N \mid K)$ and $U^{\prime}=G\left(N \mid K^{\prime}\right)$. The Galois group $G(N \mid \widetilde{K})$ is the maximal normal subgroup of $G$ contained in $U ; G(N \mid \widetilde{K})=U_{G}=\bigcap_{\sigma \in G} U^{\sigma}$.

$V$ is the subgroup of $G$ generated by $U_{G}$ and $U^{\prime}$. Since $U_{G}$ is a normal subgroup of $G$ it follows that $V=\left\{u \cdot u^{\prime} \mid u \in U_{G}, u^{\prime} \in U^{\prime}\right\}=U_{G} \cdot U^{\prime}$. For all $p \in \pi, U_{p}^{G}=U_{p}^{\prime G}$ holds and $U_{p}^{G}=V_{p}^{G}$ must be shown.

" $\subset$ ": We choose a Sylow $p$-subgroup $V_{p}$ of $V$ containing $U_{p}^{\prime}$. Then

$$
V_{p}^{G} \supset U_{p}^{\prime G}=U_{p}^{G} .
$$

" $\supset$ ": Let $\sigma=u \cdot u^{\prime} \in V=U_{G} \cdot U^{\prime}$ be of order $p^{n}$ with $\operatorname{ord}\left(u^{\prime}\right)=p^{\lambda} \cdot r$ and $\operatorname{gcd}(p, r)=1$. Then $\sigma^{r}=u_{0} \cdot u^{\prime r}$ with $u_{0} \in U_{G}$. Since $u^{\prime r} \in U^{\prime}$ has $p$-power order, we have

$$
u^{\prime r} \in U_{p}^{\prime G}=U_{p}^{G} \Rightarrow u^{\prime r}=\tau \cdot u_{1} \cdot \tau^{-1} \quad \text { with } u_{1} \in U_{p}, \tau \in G .
$$

We see

$$
\sigma^{r}=u_{0} \cdot \tau \cdot u_{1} \cdot \tau^{-1} \stackrel{u_{0} \in U_{G}}{=} \underbrace{\tau \cdot \widetilde{u}_{0} \cdot \tau^{-1}}_{=u_{0}} \cdot \tau \cdot u_{1} \cdot \tau^{-1}=\tau \cdot \widetilde{u}_{0} \cdot u_{1} \cdot \tau^{-1} \in U^{G}
$$

and conclude $\sigma^{r} \in U_{p}^{G}$. Since $p$ and $r$ are coprime, $\sigma \in U_{p}^{G}$. 
TheOREM 13. Let $K\left|k, K^{\prime}\right| k$ and $L \mid k$ be extensions of algebraic number fields and $\pi$ be a set of prime numbers.

(a) If $L \mid k$ is Galois, then

$$
K \sim_{\pi, k} K^{\prime} \Rightarrow\left\{\begin{array}{l}
K L \sim_{\pi, k} K^{\prime} L \\
K \cap L \sim_{\pi, k} K^{\prime} \cap L .
\end{array}\right.
$$

(b) If $L$ is linearly disjoint from $K$ and $K^{\prime}$ over $k$, then

$$
K \sim_{\pi, k} K^{\prime} \Rightarrow K L \sim_{\pi, L} K^{\prime} L .
$$

Proof. Let $N \mid k$ be a Galois extension containing $K, K^{\prime}$ and $L, G=$ $G(N \mid k)$ its Galois group and $U, U^{\prime}, V$ the corresponding subgroups. For $p \in \pi$ we choose Sylow $p$-subgroups.

(a) Our task is to show

$$
(U \cap V)_{p}^{G}=\left(U^{\prime} \cap V\right)_{p}^{G}
$$

and

$$
(U V)_{p}^{G}=\left(U^{\prime} V\right)_{p}^{G}
$$

For this purpose we establish

$$
(U \cap V)_{p}^{G}=U_{p}^{G} \cap V_{p}^{G} .
$$

Proof of (16). "C" is plain. " $\supset$ ": For $x=\sigma u \sigma^{-1}=\tau v \tau^{-1} \in U_{p}^{G} \cap V_{p}^{G}$ we calculate

$$
u=\sigma^{-1} \tau v \tau^{-1} \sigma \in V^{G}=V .
$$

Hence $u \in(U \cap V)_{p}^{G}$ and therefore $x \in(U \cap V)_{p}^{G}$.

(16) together with the assumption $U_{p}^{G}=U_{p}^{\prime G}$ yields the proof of (14):

$$
(U \cap V)_{p}^{G}=U_{p}^{G} \cap V_{p}^{G}=U_{p}^{\prime G} \cap V_{p}^{G}=\left(U^{\prime} \cap V\right)_{p}^{G} .
$$

Proof of (15). Let $\sigma=u v \in(U V)_{p}$ with $\operatorname{ord}(u)=p^{i} \cdot r$ and $\operatorname{gcd}(r, p)$ $=1$. As in the proof of Theorem 12 one uses the fact that $V$ is a normal subgroup of $G$ in order to prove $\sigma^{r}=u^{r} \cdot \widetilde{v}$ with $u^{r} \in U_{p}^{G}=U_{p}^{\prime G}$ and $\widetilde{v} \in V$. Hence we may write $u^{r}=\tau \cdot u^{\prime} \cdot \tau^{-1}$ with $u^{\prime} \in U_{p}^{\prime}, \tau \in G$ and see that

$$
\sigma^{r}=\tau \cdot u^{\prime} \cdot \tau^{-1} \cdot \widetilde{v}=\tau \cdot u^{\prime} \cdot \tau^{-1} \cdot \tau \cdot \widetilde{\widetilde{v}} \cdot \tau^{-1} \in\left(U^{\prime} \cdot V\right)_{p}^{G} .
$$

On the other hand, $\left\langle\sigma^{r}\right\rangle=\langle\sigma\rangle$, so that $\sigma \in\left(U^{\prime} \cdot V\right)_{p}^{G}$.

(b) In group theoretic terms our assumption means $(V: V \cap U)=$ $(G: U)$, hence $(G: V \cap U)=(G: U) \cdot(G: V)$. This is only possible if $G=U \cdot V$. Hence $U^{G}=U^{V}$ and $U^{\prime G}=U^{\prime V}$. Thus $(U \cap V)_{p}^{V}=\left(U_{p}^{G} \cap V\right)^{V}=$ $\left(U_{p}^{\prime G} \cap V\right)^{V}=\left(U^{\prime} \cap V\right)_{p}^{V}$.

We have seen so far that weakly Kronecker equivalent number fields contain the same Galois extensions $k$ and there are only finitely many minimal fields in every weak Kronecker class. We now prove that weakly Kronecker 
equivalent fields are not linearly disjoint over $k$ and thus generalize a result of N. Klingen [7]. Moreover, we show that the weak Kronecker class of the ground field consists of $k$ only; this generalizes the well known fact that the Kronecker class of the ground field is trivial.

A third important result is that given finitely many weakly Kronecker equivalent fields $K_{1}, \ldots, K_{n}$ (for example the minimal fields of a class) there is a prime $p$ which divides all degrees $\left(K_{1}: k\right), \ldots,\left(K_{n}: k\right)$. This theorem is a far reaching generalization of a result of N. Klingen [7] who showed that the degrees of two Kronecker equivalent fields, different from $k$, are not coprime.

These results may be applied to fields with "almost equal" groups of global norms.

TheOrem 14. Let $K_{1} \mid k$ be a non-trivial extension of number fields.

(a) Let $K_{1}$ and $K_{2}$ be weakly Kronecker equivalent over $k$. Then $K_{1}$ and $K_{2}$ are not linearly disjoint over $k$.

(b) Let $K_{1}, \ldots, K_{m}$ be weakly Kronecker equivalent over $k$. Then

$$
\operatorname{gcd}\left(\left(K_{1}: k\right), \ldots,\left(K_{m}: k\right)\right)>1 .
$$

Pr o of. We take a Galois extension $N \mid k$ which contains all fields under consideration. Within $G=G(N \mid k)$ we consider the corresponding subgroups $U_{1}, \ldots, U_{m}$.

(a) We assume that $K_{1}$ and $K_{2}$ are linearly disjoint and choose Sylow $p$-subgroups

$$
U_{2, p} \supset\left(U_{1} \cap U_{2}\right)_{p} .
$$

As in the proof of Theorem 13(b) we get $U_{1}^{G}=U_{1}^{U_{2}}$ and

$$
\bigcup_{p \mid \# U_{2}}\left(U_{1} \cap U_{2}\right)_{p}^{U_{2}}=\bigcup_{p \mid \# U_{2}}\left(U_{1, p}^{G} \cap U_{2}\right)^{U_{2}} .
$$

Therefore

$$
\bigcup_{p \mid \# U_{2}}\left(U_{1} \cap U_{2}\right)_{p}^{U_{2}}=\bigcup_{p \mid \# G}\left(U_{2, p}^{G} \cap U_{2}\right)^{U_{2}}=\bigcup_{p \mid \# U_{2}} U_{2, p}^{U_{2}} .
$$

By Theorem 8 and Remark 16 below we get the contradiction $U_{1} \cap U_{2}=U_{2}$.

(b) By Theorem 8 there is $\sigma \in G$ of prime power order $p^{i}$ which acts without fixed points on $G / U_{1}$. Thus $p$ divides $\left(K_{1}: k\right)$. Since $U_{i, p}^{G}=U_{j, p}^{G}$ $(i, j \in\{1, \ldots, m\}), \sigma$ also acts without fixed points on $G / U_{i}$. Hence $p$ divides all degrees $\left(K_{i}: k\right)$.

TheOREM 15. Let $k \sim_{s, k} K$. Then $K=k$. The weak Kronecker class of the ground field consists of one field only.

Proof. One can use Theorem 14(a) or (b).

REMARK 16. The following conditions are equivalent and hold by the theorem of Fein, Kantor and Schacher: 
(a) No number field $k$ is weakly Kronecker equivalent over $k$ to a nontrivial extension $K \mid k$.

(b) Every finite group $G$ acting transitively on a finite set $X$ with $\# X>1$ contains an element of prime power order, which acts without fixed points.

(c) No finite group $G$ satisfies $\bigcup_{p \mid \# G} G_{p}^{G}=\bigcup_{p \mid \# G} U_{p}^{G}$ with a subgroup $U \neq G$ of $G$.

Proof. (a) $\Leftrightarrow(\mathrm{c})$ is contained in Theorem 3 .

$(\mathrm{c}) \Rightarrow(\mathrm{b})$. Assume there is a group $G$ for which (b) does not hold and take as $U$ one of the (conjugate) point stabilizers $G_{x}$. Every $\sigma \in \bigcup_{p \mid \# G} G_{p}^{G}$ has a fixed point, hence lies in $\bigcup_{p \mid \# G} U_{p}^{G}$.

(b) $\Rightarrow$ (c). Let $(G, U)$ be a counterexample. $G$ acts transitively on the set $X=G / U$ with $\# X>1$, but every $\sigma \in \bigcup U_{p}^{G}$ has a fixed point.

6. Rigidity. The first known field with trivial Kronecker class was the ground field. W. Jehne worked on extensions of degree two of the ground field and reduced the question of whether there are fields of degree two with trivial Kronecker class to a statement about finite simple groups, which he used in order to solve the problem in some cases. Thus he conjectured that fields of degree two have trivial Kronecker class. This conjecture was finally confirmed by J. Saxl [18] after the classification of finite simple groups was complete. Other rigidity results for Kronecker classes are due to Ch. E. Praeger [15]-[17], N. Klingen [8], [9] and R. Guralnick [3]. It is only too natural to ask which weak Kronecker classes are trivial. The following theorem due to R. Guralnick [3] fits perfectly to solve the question in "almost all" (see [22]) cases. The reader should again note the application to norm groups.

Theorem 17. Let $H \subset G$ be a subgroup of a finite group $G$ with index $(G: H)=n$. If $U=H_{G}$ satisfies

$$
G / U \simeq A_{n>5} \quad \text { or } \quad G / U \simeq S_{n}
$$

then every subgroup $H^{\prime}$ of $G$ with

$$
\bigcup_{p \mid \# G} H_{p}^{\prime G} \supset \bigcup_{p \mid \# G} H_{p}^{G}
$$

contains a subgroup conjugate to $H$ in $G$.

From this and Theorem 3 we get the following very important theorem (cf. Theorem 1):

TheOREM 18. Let $K \mid k$ be of degree $n$ with $G(\widetilde{K} \mid k)=S_{n}$ or $G(\widetilde{K} \mid k)=$ $A_{n>5}$. Given an arbitrary extension $K^{\prime} \mid k$ the following statements hold:

(a) $T F(K \mid k) \stackrel{\delta}{\subset} T F\left(K^{\prime} \mid k\right) \Rightarrow K^{\prime}$ is conjugate over $k$ to $k$ or to $K$. (Here $\stackrel{\delta}{\complement}$ means "contained up to a set of Dirichlet density $0 . ")$ 
(b) If $K$ and $K^{\prime}$ are weakly Kronecker equivalent over $k$, then $K$ and $K^{\prime}$ are conjugate over $k$.

Pr o of. In primitive permutation groups $G$ point stabilizers are maximal subgroups $\neq G$.

Theorem 17 contains the theorem of Fein, Kantor and Schacher $(n=1)$ and the following extension of Saxl's result:

THEOREM 19. Weak Kronecker classes of quadratic extensions $K \mid k$ are trivial.

Proof. Take $n=2$.

The following rigidity result is originally due to L. Stern [21] (who uses quite different notions). Here we present a very convincing short proof.

THeOREM 20. Weakly Kronecker equivalent fields $K \mid k$ and $K^{\prime} \mid k$ of the same degree $(K: k)=\left(K^{\prime}: k\right) \leq 5$ are conjugate over $k$.

Proof. $n=4$ : If $\# G(\widetilde{K} \mid k)=24$ we are in the $S_{4}$-case and done by Theorem 18. $K \mid k$ and $K^{\prime} \mid k$ are extensions of prime power degree, hence they are minimal in their weak Kronecker class and thus possess the same normal closure over $k$ (Theorem 22 below and Theorem 12). If $(\widetilde{K}: k)<24$ and $K \mid k$ is not Galois, then the groups corresponding to $K$ and $K^{\prime}$ are cyclic of prime order, hence conjugate.

$n=2,3,5: K$ and $K^{\prime}$ are arithmetically equivalent over $k$ (Theorem 23) and conjugate by Perlis [14].

\section{Special weak Kronecker classes}

Theorem 21. Let $\pi$ be a set of prime numbers, and $p \in \pi$. For $\pi$ Kronecker equivalent fields $K \sim_{\pi, k} L$ where $K \mid L$ is an extension of degree $(K: L)=p$ we have

$$
p<(L: k) \text {. }
$$

Proof. Theorem 21 is an extension of [7], Satz 8. The original proof only requires some simple modifications.

TheOREM 22. Let $K \mid k$ and $K^{\prime} \mid k$ be extensions of prime power degrees $p^{i}, q^{j}\left(i, j \in \mathbb{N}_{+}\right)$and $\pi \supset\{p, q\}$ a set of prime numbers. If $K$ and $K^{\prime}$ are $\pi$-Kronecker equivalent, then

$$
(K: k)=\left(K^{\prime}: k\right) .
$$

In particular, fields of prime power degree are minimal in their $\pi$-Kronecker class.

Proof. Let $N \mid k$ be a Galois extension containing $K$ and $K^{\prime}$. Since $K$ and $K^{\prime}$ obviously are weakly Kronecker equivalent over $k$, we see that $p=q$ 
(Theorem 14). Let $G_{p}$ be a Sylow $p$-subgroup of $G(N \mid k)$ with fixed field $k_{p}$. $k_{p}$ is linearly disjoint from $K$ and $K^{\prime}$ over $k$ and Theorem 13 yields

$$
K k_{p} \sim_{\pi, k_{p}} K^{\prime} k_{p},
$$

hence

$$
K k_{p} \sim_{k_{p}} K^{\prime} k_{p}
$$

An (unpublished) result of $\mathrm{N}$. Klingen now says $\left(K k_{p}: k_{p}\right)=\left(K^{\prime} k_{p}: k_{p}\right)$. Since $\left(K k_{p}: k_{p}\right)=(K: k)$ we obtain $(K: k)=\left(K^{\prime}: k\right)$. The second assertion follows from Theorem 15 .

TheOREM 23. Let $\{p, q\} \subset \pi$ be a set of prime numbers. For $\pi$-Kronecker equivalent fields $K \mid k$ and $K^{\prime} \mid k$ of degree $(K: k)=p,\left(K^{\prime}: k\right)=q$ the following statements hold:

(a) $\left(K^{\prime}: k\right)=(K: k)$.

(b) $K$ and $K^{\prime}$ are arithmetically equivalent over $k$.

(c) If $G=G(\widetilde{K} \mid k)$ is solvable, then $K$ and $K^{\prime}$ are conjugate over $k$.

(d) The possible degrees $p$ and groups $G(\widetilde{K} \mid k)$ for which $K$ and $K^{\prime}$ are not conjugate are known by the work of $W$. Feit [2].

(e) In any case,

(i) $\mathrm{Cl}(K)_{p} \simeq \mathrm{Cl}\left(K^{\prime}\right)_{p}$.

(ii) $\bigwedge_{n \in \mathbb{N}} K_{n}\left(\mathcal{O}_{K}\right)_{p} \simeq K_{n}\left(\mathcal{O}_{K^{\prime}}\right)_{p}$. (Here $K_{n}\left(\mathcal{O}_{K}\right)_{p}$ denotes the $p$ primary part of the $n$-th Quillen K-group of the ring of integers of $K$.)

(iii) $U_{K} \simeq U_{K^{\prime}}$.

(iv) $\widetilde{K}=\widetilde{K^{\prime}}$.

Proof. For extensions $K\left|k, K^{\prime}\right| k$ of prime degree $p$ the following conditions are equivalent:

(i) $K$ and $K^{\prime}$ are arithmetically equivalent over $k$.

(ii) $\widetilde{K}=\widetilde{K^{\prime}}$.

(iii) $K$ and $K^{\prime}$ are not linearly disjoint over $k$.

Indeed, (i) $\Rightarrow$ (ii) is obvious. (ii) $\Rightarrow\left(\right.$ iii): $p^{2}$ does not divide $p$ !. (iii) $\Rightarrow($ i): Since the Sylow $p$-subgroups of $U$ and $U^{\prime}$ are conjugate (i) follows from [4].

Now by Theorem 22, (a) follows. From Theorem 12 we conclude $\widetilde{K}=\widetilde{K^{\prime}}$, hence (b).

(c) $U$ and $U^{\prime}$ are $\{p\}^{\prime}$-Hall subgroups.

(d) See $[2]$.

(e) (ii) comes from Komatsu [10] and contains (i). (iii) is known by [14].

As we have seen, weakly Kronecker equivalent fields of prime degree have the same normal closure over $k$. In general this is false. As an example we may take $A_{4}$-extensions $N \mid k$ of number fields. Here the cyclic-cubic subfield 
and every of the subfields of degree 6 are (weakly) Kronecker equivalent but do not have the same normal closure. The theorem holds, however, if $K$ and $K^{\prime}$ can be embedded in a nilpotent extension $N \mid k$ (Stern [21]). In this case $K$ and $K^{\prime}$ are Kronecker equivalent, since nilpotent groups are direct products of their Sylow $p$-subgroups. If in addition there is no prime $p$ so that $p^{4}$ divides $(N: k)$ a simple consideration shows that $K$ and $K^{\prime}$ are conjugate. Further, one has

TheOREM 24. Let $K$ and $K^{\prime}$ be weakly Kronecker equivalent over $k$. If there is a nilpotent extension $N \mid k$ containing $K$ and $K^{\prime}$ then the degrees $(K: k)$ and $\left(K^{\prime}: k\right)$ possess the same prime divisors.

Proof. Let $p \nmid\left(K^{\prime}: k\right)$ be a prime number. Since the Sylow $p$-subgroup $G_{p}$ of $G(N \mid k)$ is normal in $G$ we have

$$
G_{p}=U_{p}^{\prime}=U_{p}^{\prime G}=U_{p}^{\prime G_{p}}=U_{p}^{G_{p}}
$$

hence $U_{p}=G_{p}$.

In general one can only prove that $(N: K)$ and $\left(N: K^{\prime}\right)$ possess the same prime divisors.

EXAMPLE 25. Let $K \mid k$ be an extension with normal closure $N=K L$ and $L \mid k$ abelian. Given a set $\pi$ of prime numbers which contains all divisors of $(N: k)$ the following statements hold:

(a) $K$ is minimal in its $\pi$-Kronecker class.

(b) Every subfield $K^{\prime}$ of $N \mid k$ with $K^{\prime} \sim_{\pi, k} K$ is also minimal and satisfies

$$
\left(K^{\prime}: k\right)=(K: k) \quad \text { and } \quad \widehat{\Psi}_{U}=\widehat{\Psi}_{U^{\prime}} .
$$

Further, $U^{\prime}=G\left(N \mid K^{\prime}\right)$ is a complement to $H=G(N \mid L)$ in $G_{0}=$ $U H \triangleleft G$, e.g. $U H=U^{\prime} H$ and $U^{\prime} \cap H=1$.

(c) Every extension $K^{\prime} \mid k$ with $\left(K^{\prime}: k\right)=(K: k)$ and $K^{\prime} \sim_{\pi, k} K$ is minimal and contained in $N$.

(d) As an example one may take $k(\sqrt[n]{a})=K$ with $k\left(\sqrt[n]{a}, \zeta_{n}\right)=N$ and $k\left(\zeta_{n}\right)=L$.

Proof. Let $K^{\prime}$ be a subfield of $N \mid k$ with $K^{\prime} \sim_{\pi, k} K$. Then for all $p \in \pi$, $\Phi_{U_{p}}=\Phi_{U_{p}^{\prime}}([7])$. Hence $\widehat{\Psi}_{U}=\widehat{\Psi}_{U^{\prime}}$. Thus $(K: k)=\left(K^{\prime}: k\right)$ (remark after Theorem 13) and $\widetilde{K}=\widetilde{K}^{\prime}=K L$. In group theoretic terms $N=K L$ means $U \cap H=1$. $U^{\prime} \cap H \neq 1$ would for at least one $p \in \pi$ imply $U_{p}^{\prime} \cap H \neq 1$. But this together with $U_{p}^{G}=U_{p}^{\prime G}$ and $H \triangleleft G$ yields the contradiction $U \cap H \neq 1$.

$U^{\prime} H$ corresponds to $K^{\prime} \cap L=K \cap L$, thus $U^{\prime} H=U H$.

An arbitrary field $K^{\prime}$ with $K^{\prime} \sim_{\pi, k} K$ and $(K: k)=\left(K^{\prime}: k\right)$ contains a minimal field. (c) follows, because all minimal fields are contained in $N$ and have degree $(K: k)$. 


\section{References}

[1] B. Fein, W. Kantor and M. Schacher, Relative Brauer groups II, J. Reine Angew. Math. 328 (1981), 374-403.

[2] W. Feit, Some consequences of the classification of finite simple groups, in: Proc. Sympos. Pure Math. 37, Amer. Math. Soc., 1980, 175-181.

[3] R. Guralnick, Zeroes of permutation characters with applications to prime splitting and Brauer groups, J. Algebra 131 (1990), 294-302.

[4] R. Guralnick and D. Wales, Subgroups inducing the same permutation representation II, ibid. 96 (1985), 94-113.

[5] W. Jehne, Kronecker classes of algebraic number fields, J. Number Theory 9 (1977), 279-320.

[6] - On Kronecker classes of atomic extensions, Proc. London Math. Soc. (3) 34 (1977), 32-64.

[7] N. Klingen, Zahlkörper mit gleicher Primzerlegung, J. Reine Angew. Math. 299/300 (1978), 342-384.

[8] - Atomare Kronecker-Klassen mit speziellen Galoisgruppen, Abh. Math. Sem. Univ. Hamburg (1979), 42-53.

[9] - Rigidity of decomposition laws and number fields, J. Austral. Math. Soc. Ser. A 51 (1991), 171-186.

[10] K. Komatsu, Einige Bemerkungen über Dedekindsche Zetafunktionen und KGruppe, Arch. Math. (Basel) 54 (1990), 164-165.

[11] M. Lochter, Neue zahlentheoretische Aspekte der Kronecker-Äquivalenz, thesis, Köln, 1992.

[12] - New characterizations of Kronecker equivalence, J. Number Theory, to appear.

[13] J. Neukirch, Class Field Theory, Springer, 1986.

[14] R. Perlis, On the equation $\zeta_{K}(s)=\zeta_{K^{\prime}}(s)$, J. Number Theory 9 (1977), 342-360.

[15] Ch. E. Praeger, Covering subgroups of groups and Kronecker classes of fields, J. Algebra 118 (1988), 455-463.

[16] - On octic field extensions and a problem in group theory, in: Group Theory, Proceedings of the 1987 Singapore Conference, Walter de Gruyter, Berlin, 1989, 443-463.

[17] - Kronecker classes of field extensions of small degree, J. Austral. Math. Soc. Ser. A 50 (1991), 297-315.

[18] J. Saxl, On a question of W. Jehne concerning covering subgroups of groups and Kronecker classes of fields, J. London Math. Soc. (2) 38 (1988), 243-249.

[19] V. Schulze, Kronecker-äquivalente Körpererweiterungen und p-Ränge, J. Reine Angew. Math. 328 (1981), 9-21.

[20] J. P. Serre, Linear Representations of Finite Groups, Springer, Berlin, 1977.

[21] L. Stern, On the equality of norm groups of global fields, J. Number Theory 36 (1990), 108-126.

[22] B. L. van der Waerden, Die Seltenheit der Gleichungen mit Affekt, Math. Ann. 109 (1934), 13-16.

FB 9, MATHEMATIK

UNIVERSITÄT DES SAARLANDES

66041 SAARBRÜCKEN, GERMANY

Received on 7.8 .1992

and in revised form on 1.12.1992 